\title{
Prediction of one-repetition maximum from submaximal ratings of perceived exertion in older adults pre- and post-training
}

\author{
François-Denis Desgorces • Rémi Thomasson • \\ Shehrazad Aboueb · Jean-François Toussaint • \\ Philippe Noirez
}

Received: 17 December 2014/ Accepted: 12 February 2015

(C) Springer International Publishing Switzerland 2015
F.-D. Desgorces $(\bowtie) \cdot$ R. Thomasson · S. Aboueb .

J.-F. Toussaint $\cdot$ P. Noirez

Institut de Recherche bioMédicale et d'Epidemiologie du Sport (IRMES), Institut National du Sport de l'Expertise et de la Performance (INSEP), Paris, France

e-mail: francois.desgorces@ parisdescartes.fr

F.-D. Desgorces · R. Thomasson · J.-F. Toussaint · P. Noirez Université Paris Descartes, Sorbonne Paris Cité, EA 7329, Paris, France

R. Thomasson

Université Paris Est, Marne-la-Vallée, France

S. Aboueb

Collegium of Sciences and Technology, Université d'Orléans, Orléans, France

J.-F. Toussaint

Centre d'Investigation en médecine du Sport, Hôtel-Dieu, Assistance Publique-Hôpitaux de Paris, Paris, France
That RPE associated to its corresponding load was subjected to a linear regression analysis to extrapolate the maximal RPE score and its corresponding 1-RM.

Results RPE and relative intensities of sets appeared related pre- $\left[r^{2}=0.59\right.$, standard error of estimate $(\mathrm{SEE})=13.3 \%] \quad$ and $\quad$ post-training $\quad\left(r^{2}=0.83\right.$, SEE $=8.1 \%)$. Differences between measured and predicted 1-RM were reduced from the beginning to the end of training but standard deviations remained high $(17.4 \pm 11.8$ vs. $4.2 \pm 11.1 \mathrm{~kg})$. Pre-training, $1-\mathrm{RM}$ expressed relatively to body weight was negatively related with the errors of 1-RM predictions $\left(r^{2}=0.39, p=0.03\right)$. Conclusions In older subjects, RPE may be used to predict 1-RM; however, the predicted value deviates considerably from the measured one, necessitating cautious application. Importantly, this method allows to capture training-induced change in 1-RM, thus making possible assessing training's effectiveness and allowing its modification if necessary.

Keywords Strength training - Aging - Perceived exertion · One-repetition maximum $\cdot$ Rehabilitation

\section{Introduction}

Aging is associated with decreased strength and muscle mass that is defined as sarcopenia [1]. Strength training in the elderly increases protein synthesis, muscle mass and strength, and improves locomotor functions such as walking speeds and postural control [2, 3]. As a consequence, strength training in rehabilitation program for older adults is largely promoted [4].

Peterson et al. [5] suggested that, similar to that observed in healthy young individuals, higher intensities in 
resistance exercises for older patients provide best strength improvements. Therefore, individual's one-repetition maximum (1-RM) is required to calculate and prescribe intensity for resistance training, while 1-RM testing protocols may enhance the risk of injuries and are time-consuming. Obviously, these limitations of 1-RM testing may be enhanced in elderly $[6,7]$.

In training and rehabilitation fields, 1-RM is frequently predicted using submaximal tests and applications of equations for 1-RM prediction [8]. The accuracy of these equations has been linked to specific exercises, to the populations assessed and to the relative strength used for repetition maximum testing $[8,9]$. Some studies reported that the use of such prediction equations for older adults may be valid for 1-RM estimate although errors of estimate may appear slightly high [6, 10, 11]. Furthermore, direct as well as indirect assessment of 1-RM require participants to perform repetitions until muscular failure which enhance muscular and cardiovascular health risks in untrained individuals and particularly elderly [6]. To avoid risks associated with tests performed until exhaustion in population at risk, including older adults, some authors supported the use of ratings of perceived exertion (RPE) during graded exercises to predict maximal oxygen consumption [12-14]. A similar use of RPE to predict 1-RM was reported as providing highly accurate estimates although the loads to lift were presented in a random order and was not apparent to subjects [15]. Older adults' ability to discriminate the weight of lifted object may appear reduced and their use of RPE for 1-RM prediction remains to be tested [16]. Notwithstanding, according to the reported training state influence on RPE, a rehabilitation training program for the elderly could increase the subject's ability in RPE use as suggested previously [17].

The protocol used in this study was similar to that proposed by Eston and Evans [15] using light to moderately heavy loads and a very low repetition number during resistance exercise sets. The aim of the present study was to assess the accuracy of 1-RM prediction from RPE of resistance exercises performed in submaximal sets in older adults at the beginning and the end of a 12-week strength training program.

\section{Materials and methods}

\section{Subjects}

Eighteen subjects $(70.4 \pm 4.5$ years $)$ volunteered to participate in the present study after being informed of the nature of the experiments. Each subject signed a consent form that outlined possible risks due to the procedure. The protocol was approved by the local Ethics Committee. None of the subjects had any background in regular strength and/or endurance training or competitive sports of any kind in the previous 10 years. All lived at home and were able to perform activities of natural daily life independently. Subjects were required to have medical approval before participating in the study. No medication was being taken by the subjects that would have been expected to affect physical performance.

Study design

Before the first session and at the end of the 12-week program, subjects heights were measured with a height measurement scale to the nearest $\mathrm{cm}$, weight to the nearest $0.1 \mathrm{~kg}$ and body composition by an impedance weight scale (Tanita, SC-331). To decrease higher risk of injury occurring during the first weeks of strength training, subjects began by performing four sessions of familiarization to strength training and exercises during 2 weeks $[18,19]$. Training sessions, as well as those dedicated to study measures, were all separated by at least $72 \mathrm{~h}$.

The training program consisted of a total of $21.6 \pm 2.2$ training sessions over 12 weeks. The program was composed of exercises combining endurance (cycling, stairs climbing and rowing ergometers; $20-30$ min per session) and strength training exercises. Strength training was composed of varied exercises (horizontal leg press, knee extension, knee flexion, chest press, seated row, elbow extension, and elbow flexion), intensities (from 40 to $80 \%$ of 1-RM) and volumes (approximately, from 20 to 2 repetitions within 2-4 sets).

Subjects were tested during the $3 \mathrm{rd}$ and 12 th weeks of the training rehabilitation program to determine their abilities to use RPE for 1-RM prediction in horizontal leg press (Technogym, Issy les Moulineaux, France) using a method described by Eston and Evans [15]. Horizontal leg press was chosen because it allows reducing the influence of technical abilities on 1-RM and the movement appeared very safe for subjects.

Session for 1-RM direct assessment and submaximal sets

Individual 1-RM on horizontal leg press was assessed at the end of 2nd and 11th training weeks. Subjects completed a light standardized warm-up as described by Kraemer and Fry [20] (a first set of 10 repetitions at an easy load followed by $1 \mathrm{~min}$ rest and then four repetitions at moderately heavy loads). Thereafter, subjects performed 3-5 attempts to reach 1-RM on the horizontal leg press (to the nearest $5 \mathrm{~kg}$ ) with $5 \mathrm{~min}$ of rest period between each attempt (only one repetition performed in each attempt).

The sessions for submaximal sets completion and corresponding RPE records followed the protocol reported by 
Eston and Evans [15]. In the 3rd and 12th training weeks, after the same warm-up described for direct 1-RM assessment, participants performed three sets of two repetitions on horizontal leg press; the sets were performed at a pre-determined intensity (approximately, $20 \%$ of 1-RM:Int20, $45 \%$ of 1-RM:Int45 and $70 \%$ of 1-RM:Int70). The three loads were randomly presented to the participant and were applied without making them apparent to the participant. The RPE was recorded following each of these submaximal sets.

Ratings of perceived exertion

The RPE was measured with the Borg Category Ratio scale (CR-10; from 0 to 10) [21], as previously realized during strength training studies [22], without numerical rating of 0.5 and using the categorical ratings from "no exertion at all" to "maximal exertion" to facilitate the subjects' ability to appreciate the effort involved.

The CR-10 scale was explained to subjects at the beginning of the 2nd training week and was used during submaximal sets sessions (3rd and 12th training weeks): "You are about to undergo a weight lifting exercise test. The scale before you contains numbers from 0 to 10 and will be used to assess your perceptions of exertion while lifting these weights. The perception of physical exertion is defined as the subjective strain, discomfort, and/or fatigue that you feel during exercise. We use this scale so that you may translate into numbers your feelings of exertion while you exercise. The numbers on the scale represent a range of feelings from no exertion at all (0) to maximal exertion (10)".

Data analysis

The 1-RM predictions were calculated by entering individual participant RPE values and load $(\mathrm{kg})$ from each submaximal set into a linear regression analysis that provided individual regression equation to extrapolate a theoretical 1-RM at CR-10 of 10 [15].

Linear regression was used to determine the relationship between the RPE and the relative intensities of all sets (1-RM percentage) pre- and post-training. Using pre- and post-training results, relationships between 1-RM errors of prediction and subjects' characteristics (absolute 1-RM, 1-RM increases, 1-RM expressed relative to body weight, age, weight, body mass index, fat-free mass) were analyzed by linear regression to identify the possible origins of load misperception. One-way analyses of variance for repeated measures were performed to detect differences between RPE, predicted and measured 1-RM in pre- and posttraining periods. If a main effect was observed, the post hoc Tukey's test was applied to determine which condition provided differences. The accuracy of the 1-RM prediction was quantified by the explained variance $\left(r^{2}\right)$ of the relationship between measured and predicted 1-RM and by the standard error of estimate (SEE). Statistical significance was accepted at $p<0.05$ (two-sided for $t$ tests). Data in the text are given as mean \pm standard deviation (SD). $R$ software (version 3.0.2, R Foundation for Statistical Computing, Vienna, Austria) was used for the analysis.

\section{Results}

Each subject's weight was unchanged post-training $(p=0.08)$ but fat-free mass was slightly increased after 12 weeks of training $(p=0.04)$; subjects' characteristics and 1-RM are presented in Table 1. Relative intensities of submaximal sets and RPE recorded following these sets are presented in Table 2. At the beginning and the end of the period, RPE significantly differed between Int20, Int45 and Int70 ( $p \leq 0.001)$ and after training, post-training RPE was reduced at Int20 and Int45 sets (respectively, $p=0.01$ and $p=0.02)$. Measured 1-RM was increased after 12 weeks of training ( $p=0.001)$. RPE and relative intensities of sets (1-RM percentage) were related before training $\left(r^{2}=0.59\right.$, SEE $=13.3 \%) \quad$ and $\quad$ post-training $\quad\left(r^{2}=0.83\right.$, SEE $=8.1 \%)$. When the analyses were conducted separately for each intensity (i.e., Int20, Int45 and Int70), the relationships were not significant $(p>0.05)$. Measured and predicted 1-RM values differed at the beginning $(110.5 \pm 8.8$ vs. $93.1 \pm 15.9, p=0.001)$ but not at the end of the training period $(132.2 \pm 10.1$ vs. $127.6 \pm 18.6$, $p=0.07)$. The mean prediction errors at the beginning of the study appeared high $(17.4 \pm 11.8 \mathrm{~kg})$ and were reduced after training $(4.6 \pm 11.1 \mathrm{~kg})$ but standard deviations remained elevated. Relationships between measured and predicted 1-RM values appeared slightly improved by training (respectively, $r^{2}=0.45$, SEE $=10.2$ vs. $r^{2}=0.52$, SEE $=8.2 \mathrm{~kg}$; Fig. 1). Pre-training, 1-RM expressed relative to body weight was negatively related with errors of 1 -RM predictions $\left(r^{2}=0.39, p=0.03\right.$; Fig. 2); this relationship was not significant post-training $(p=0.1)$ and no other relationship appeared between

Table 1 Subjects' anthropometrical characteristics and leg-press one-repetition maximum (1-RM) pre- and post-training

\begin{tabular}{ccll}
\hline Weight $(\mathrm{kg})$ & Height $(\mathrm{kg})$ & $\begin{array}{l}\text { Fat-free } \\
\text { mass }(\%)\end{array}$ & $\begin{array}{l}\text { Measured } \\
\text { 1-RM }(\mathrm{kg})\end{array}$ \\
\hline $\begin{array}{l}\text { Pre-training } \\
74.8 \pm 7.9\end{array}$ & $170.9 \pm 4.3$ & $56.1 \pm 6.4$ & $110.5 \pm 8.8$ \\
$\begin{array}{r}\text { Post-training } \\
74.9 \pm 6.9\end{array}$ & & $57.6 \pm 6.3^{*}$ & $132.2 \pm 10.1^{*}$
\end{tabular}

* Significantly different to the pre-training measures 
Table 2 Pre- and post-training recorded RPE, relative intensities used in submaximal sets (Int20 about $20 \%$ of 1-RM, Int 45 about $45 \%$ of $1-\mathrm{RM}$ and Int70 about $70 \%$ of $1-\mathrm{RM}$ )

\begin{tabular}{|c|c|c|c|c|c|}
\hline \multicolumn{2}{|l|}{ Int20 } & \multicolumn{2}{|l|}{ Int45 } & \multicolumn{2}{|l|}{ Int70 } \\
\hline 1-RM (\%) & RPE & 1-RM (\%) & RPE & 1-RM (\%) & RPE \\
\hline \multicolumn{6}{|l|}{ Pre-training } \\
\hline $21.3 \pm 2.3$ & $4.5 \pm 1.0^{\S}$ & $46.5 \pm 4.1$ & $6.7 \pm 1.3^{\mathrm{a} s}$ & $70.9 \pm 4.0$ & $8.0 \pm 0.9^{\text {a\# }}$ \\
\hline \multicolumn{6}{|l|}{ Post-training } \\
\hline $23.1 \pm 1.3$ & $3.1 \pm 0.9 * \$$ & $46.4 \pm 2.9$ & $5.3 \pm 1.0^{* \mathrm{a} \S}$ & $71.4 \pm 2.5$ & $7.4 \pm 0.8^{\mathrm{a} \#}$ \\
\hline
\end{tabular}

* Significantly different to the pre-training measures

"Significantly different to Int20

\# Significantly different to Int 45

$\S$ Significantly different to Int70

1-RM prediction errors and subjects' characteristics $(p>0.05)$

\section{Discussion}

The present study was the first to demonstrate an older adult's ability to discriminate the intensity of strength exercises by using RPE. The prediction of 1-RM from RPE although statistically significant presents high individual prediction errors suggesting a careful use of loads determined by such a method in older adults.

Pre and post-training, RPE appears related to the relative intensities of submaximal sets that are in accordance with the reported ability of RPE to describe the intensity of resistance exercises in youths and healthy individuals [23, 24]. To our knowledge, this study is the first to show that RPE could be used to identify intensities of resistance exercises in older adults although some studies reported a possible use in endurance exercise [25-27]. The relationship between RPE and set intensities requires considering the three relative intensities performed (Int20, Int45 and Int70). Previously, the ability of older adults to discriminate the weight of the lifted objects has been reported to be reduced when compared to younger adults, whereas their ability to perceive weight ratio (weight difference between two objects) may be preserved [28]. RPE provided by our subjects differed for sets performed at Int20, Int 45 and Int70 underlining the subjects' capacity to discriminate objects weights. Therefore, our results suggest that RPE of a unique set may be insufficient to accurately identify the relative load that the subject lifted, while the discrimination of several loads improves the relationship between exercise intensity and RPE. This result supports the construct of an individual RPE regression equation to estimate 1-RM as proposed by Eston and Evans [15]. When this is performed, our results show that RPE from submaximal sets can be used to provide a quite good estimation of 1-RM.
Nevertheless the statistical significance of our results, individual error of prediction as well as SEE of regression analyses appears high in both pre- and post-training sessions. Prediction errors previously reported by Eston and Evans [15] were about few kilograms, but in our results when expressed in percentage of 1-RM, they are higher than $10 \%$. These errors of prediction could be assumed as quite high for older populations according to the health perspective of rehabilitation programs.

The origins of load misperception are difficult to identify and should be linked to several factors. However, the negative relationship between errors of prediction and 1-RM expressed relative to body weight suggested that subjects with the lower relative strength were the ones presenting the lower ability for load perception. The influence of training state and fitness on RPE ability to describe endurance physical effort has already been demonstrated [29, 30]. We previously reported that, in strength training, the relationship between relative exercise volumes and RPE was enhanced in trained individuals [22]. The relationship between errors of prediction and 1-RM relative to body weight strength observed during pretraining was not significant during post-training suggesting that strength training could alter loads misperception particularly in weaker subjects. Until the present study, such training effect on the accuracy of RPE use by older adults has mainly been suggested rather than demonstrated [17].

However, the 12-week rehabilitation program results not only increase the 1-RM but also improved the ability to discriminate strength levels. Explained variance of the relationships and SEE demonstrate a higher accuracy for post-training 1-RM estimates than for pre-training estimates. For similar relative intensities, sets of RPE at Int20 and Int45 were higher in pre-training than in post-training underlining over-estimation of light loads at the beginning of the training period. This over-estimation of the strength in older adults that required to lift or grip objects has been previously reported $[11,31]$. Moreover, after recent hip 

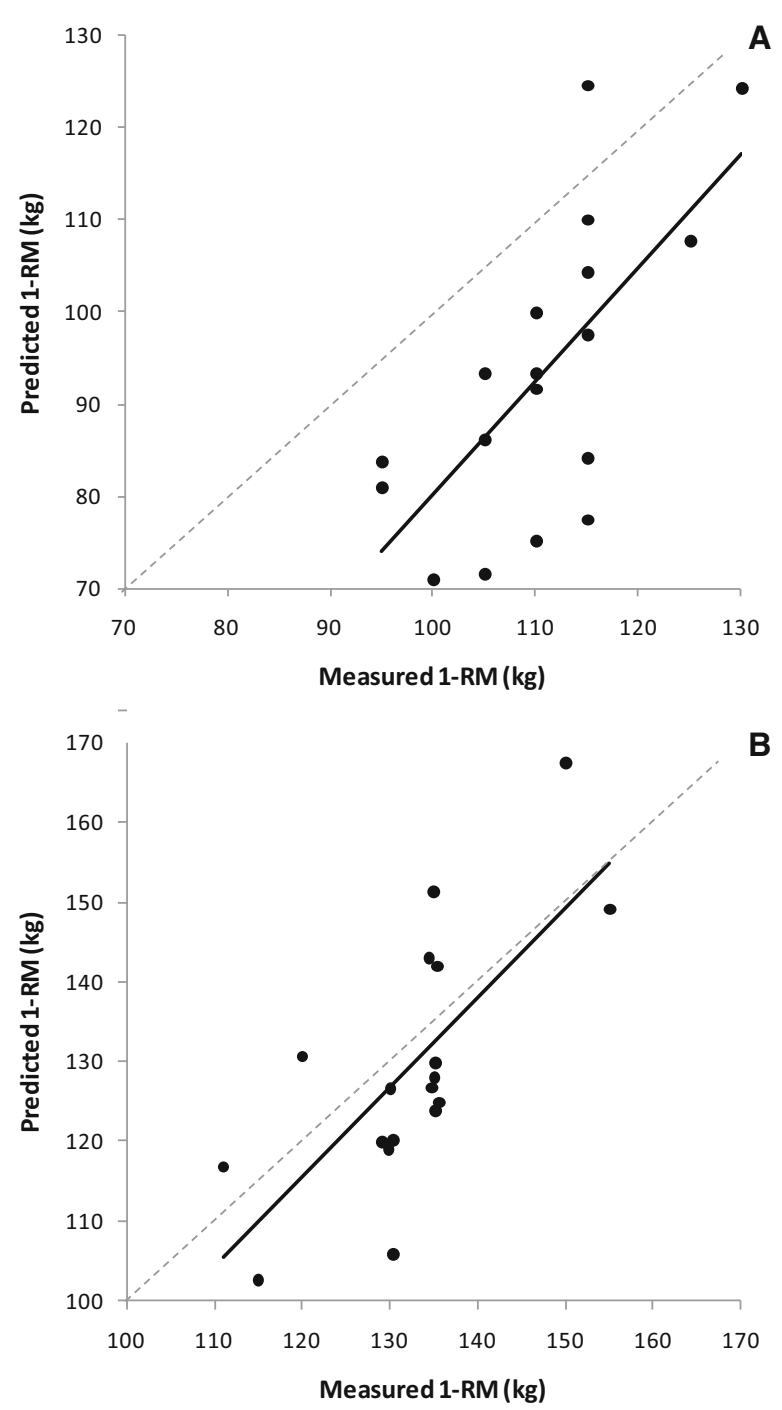

Fig. 1 Relationship between measured and predicted 1-RM from RPE of submaximal sets pre- (a) and post-training (b). Regression line in continuous black line $\left(r^{2}=0.45, p=0.02\right.$ for $\mathbf{a}$ and $r^{2}=0.52, p=0.01$ for $\mathbf{b}$ and dashed gray line for identity line

arthroplasty in older patients, a period of habituation to exercise has been proved necessary to observe a significant relationship between RPE and heart rate when exercising [26]. Such delay for an accurate RPE use could be linked to age but also to patients' pathology [32, 33]. Post-training, differences between measured and predicted 1-RM appear more balanced with under- and over-estimation of 1-RM. This 1-RM over-estimation observed during post-training may enhance the muscular risks associated with exercise. Therefore, at the beginning of training, RPE provides under-estimations of 1-RM rendering low accuracy for 1-RM predictions but remaining safe. Conversely, the higher prediction accuracy observed during post-training was

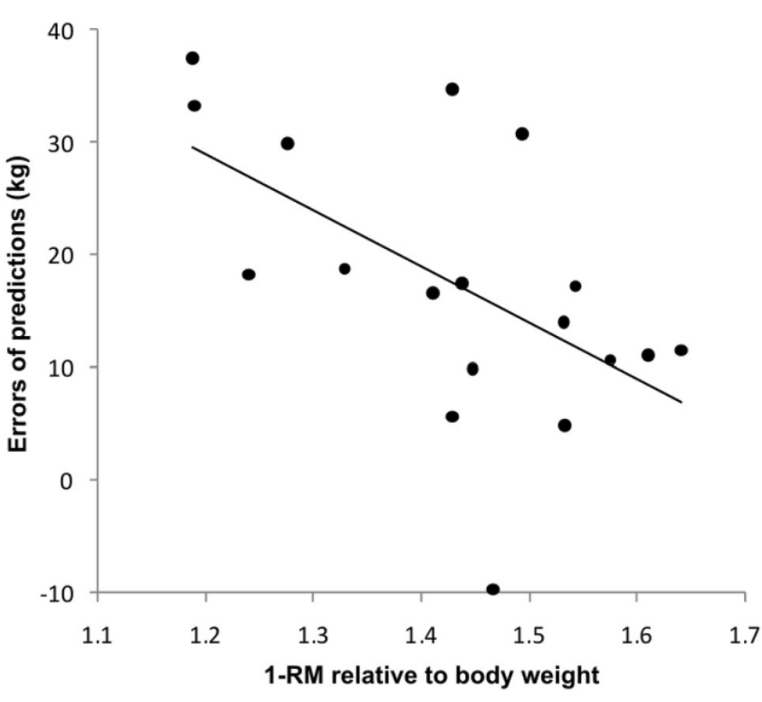

Fig. 2 Relationship between errors of prediction and one-repetition maximum (1-RM) expressed relative to body weight pre-training. Regression line in continuous black line $\left(r^{2}=0.39, p=0.03\right)$

combined with over-estimation that could be associated with enhanced potential risks for older subjects. It could be hypothesized that in pre-training, over-estimating loads may have a protective role against risks associated with physical tasks in older subjects.

We assumed that the protocol, used in the present study, cannot be applied in practice as relative loads of submaximal sets cannot be known. Our results demonstrate that $1-\mathrm{RM}$ predictions could be calculated by recording RPE after several sets of resistance exercises performed at light to moderate loads and with low repetition number. A practical method of 1-RM estimation from RPE should correspond to ratings of unknown light to moderate loads. To preserve older subject from any risks, testing loads should be modified according to previous rating to avoid high loads (i.e., maximal rating about 7-8 in CR-10 scale). After 1-RM estimation and loads determination, loads to lift should be tested by subjects before being included in the sets of strength training programs. Furthermore, it is of importance to note that enhanced strength capacities were identified by RPE underlining the fact that RPE may be an easy tool for the assessment of training effects or even for individual training monitoring in a day or week basis as proposed for young adults and athletes [34].

\section{Conclusion}

Despite aging negative effects, the present study reported that in the elderly, RPE may be used to estimate 1-RM with fairly reasonable accuracy despite using sub-maximal loads 
and only two repetitions. Pre-training, the 1-RM is rather underestimated, especially in weaker subjects, than overestimated thus rendering the method safe. In addition, RPE also allow detecting training-induced increase in 1-RM. The present method paves the way to a practical method where 1-RM will be predicted neither with prior knowledge, nor with later determination of actual 1-RM.

Conflict of interest On behalf of all authors, the corresponding author states that there is no conflict of interest.

Human and Animal Rights All procedures performed in studies involving human participants were in accordance with the ethical standards of the institutional and/or national research committee and with the 1964 Helsinki declaration and its later amendments or comparable ethical standards. This article does not contain any studieswith animals performed by any of the author.

Informed consent Informed consent was obtained from all individual participants included in the study.

\section{References}

1. Baumgartner RN, Koehler KM, Gallagher D et al (1998) Epidemiology of sarcopenia among the elderly in New Mexico. Am J Epidemiol 147:755-763

2. Fiatarone MA, O'Neill EF, Ryan ND et al (1994) Exercise training and nutritional supplementation for physical frailty in very elderly people. N Engl J Med 330:1769-1775. doi:10.1056/ NEJM199406233302501

3. Hunter GR, McCarthy JP, Bamman MM (2004) Effects of resistance training on older adults. Sports Med 34:329-348

4. Chodzko-Zajko WJ, Proctor DN, American College of Sports Medicine et al (2009) American College of Sports Medicine position stand. exercise and physical activity for older adults. Med Sci Sports Exerc 41:1510-1530. doi:10.1249/MSS. 0b013e3181a0c95c

5. Peterson MD, Rhea MR, Sen A, Gordon PM (2010) Resistance exercise for muscular strength in older adults: a meta-analysis. Ageing Res Rev 9:226-237. doi:10.1016/j.arr.2010.03.004

6. Niewiadomski W, Laskowska D, Gasiorowaska A et al (2008) Determination and prediction of one repetition maximum (1RM): safety considerations. J Hum Kinet 19:109-120

7. Ploutz-Snyder LL, Giamis EL (2001) Orientation and familiarization to $1 \mathrm{RM}$ strength testing in old and young women. J Strength Cond Res 15:519-523

8. Mayhew JL, Prinster JL, Ware JS et al (1995) Muscular endurance repetitions to predict bench press strength in men of different training levels. J Sports Med Phys Fitness 35:108-113

9. Reynolds JM, Gordon TJ, Robergs RA (2006) Prediction of one repetition maximum strength from multiple repetition maximum testing and anthropometry. J Strength Cond Res 20:584-592. doi:10.1519/R-15304.1

10. Wood TM, Maddalozzo GF, Harter RA (2002) Accuracy of seven equations for predicting 1-RM performance of apparently healthy, sedentary older adults. Meas Phys Educ Exerc Sci 6:67-94

11. Knutzen KM, Brilla LR, Caine D (1999) Validity of 1RM prediction equations for older adults. J Strength Cond Res 13:242-246

12. Coquart JBJ, Eston RG, Grosbois J-M et al (2010) Prediction of peak oxygen uptake from age and power output at RPE 15 in obese women. Eur J Appl Physiol 110:645-649. doi:10.1007/ s00421-010-1524-5

13. Al-Rahamneh HQ, Eston RG (2011) The validity of predicting peak oxygen uptake from a perceptually guided graded exercise test during arm exercise in paraplegic individuals. Spinal Cord 49:430-434. doi:10.1038/sc.2010.139

14. Smith AE, Eston RG, Norton B, Parfitt G (2014) A perceptuallyregulated exercise test predicts peak oxygen uptake in older active adults. J Aging Phys Act. doi:10.1123/japa.2013-0213

15. Eston R, Evans HJL (2009) The validity of submaximal ratings of perceived exertion to predict one repetition maximum. J Sports Sci Med 8:567-573

16. Holmin JS, Norman JF (2012) Aging and weight-ratio perception. PLoS One 7:e47701. doi:10.1371/journal.pone.0047701

17. Groslambert A, Mahon AD (2006) Perceived exertion : influence of age and cognitive development. Sports Med 36:911-928

18. Hunter GR, Wetzstein CJ, McLafferty CL Jr et al (2001) Highresistance versus variable-resistance training in older adults. Med Sci Sports Exerc 33:1759-1764

19. Shaw CE, McCully KK, Posner JD (1995) Injuries during the one repetition maximum assessment in the elderly. J Cardpulm Rehabil 15:283-287

20. Kraemer WJ, Fry AC (1995) Strength testing: development and evaluation of methodology. In: Maud P, Nieman DC (eds) Fitness and sports medicine: A health-related approach. Bull Publishing, Palo Alto

21. Borg G (1998) Perceived exertion and pain scales. Human Kinetics, Champaign

22. Testa M, Noakes TD, Desgorces F-D (2012) Training state improves the relationship between rating of perceived exertion and relative exercise volume during resistance exercises. J Strength Cond Res Natl Strength Cond Assoc 26:2990-2996. doi:10.1519/ JSC.0b013e31824301d1

23. Lagally KM, Robertson RJ, Gallagher KI et al (2002) Perceived exertion, electromyography, and blood lactate during acute bouts of resistance exercise. Med Sci Sports Exerc 34:552-559 (discussion 560)

24. Gearhart RF Jr, Goss FL, Lagally KM et al (2002) Ratings of perceived exertion in active muscle during high-intensity and low-intensity resistance exercise. J Strength Cond Res 16:87-91

25. Groslambert A, Grange CC, Perrey S et al (2006) Effects of aging on perceived exertion and pain during arm cranking in women 70 to 80 YEARS OLD. J Sports Sci Med 5:208-214

26. Grange CC, Maire J, Groslambert A et al (2004) Perceived exertion and rehabilitation with arm crank in elderly patients after total hip arthroplasty: a preliminary study. J Rehabil Res Dev 41:611-620

27. Pandolf KB, Burse RL, Goldman RF (1975) Differentiated ratings of perceived exertion during physical conditioning of older individuals using leg-weight loading. Percept Mot Skills 40:563-574

28. Norman JF, Norman HF, Swindle JM et al (2009) Aging and the discrimination of object weight. Perception 38:1347-1354

29. Micklewright D, Papadopoulou E, Swart J, Noakes T (2010) Previous experience influences pacing during $20 \mathrm{~km}$ time trial cycling. Br J Sports Med 44:952-960. doi:10.1136/bjsm.2009. 057315

30. Garcin M, Mille-Hamard L, Billat V (2004) Influence of aerobic fitness level on measured and estimated perceived exertion during exhausting runs. Int J Sports Med 25:270-277. doi:10.1055/s2004-819939

31. Parikh PJ, Cole KJ (2012) Handling objects in old age: forces and moments acting on the object. J Appl Physiol (1985) 112:1095-1104. doi:10.1152/japplphysiol.01385.2011 
32. Vlaeyen JW, Kole-Snijders AM, Boeren RG, van Eek H (1995) Fear of movement/(re)injury in chronic low back pain and its relation to behavioral performance. Pain 62:363-372

33. Ben Belgith A, Ahmaidi S, Maille P et al (2012) Quantification de la charge d'entraînement imposée au footballeur professionnel en phase de réhabilitation athlétique post-blessure. Sci Sports 27:169-174

34. Eston R (2012) Use of ratings of perceived exertion in sports. Int J Sports Physiol Perform 7:175-182 